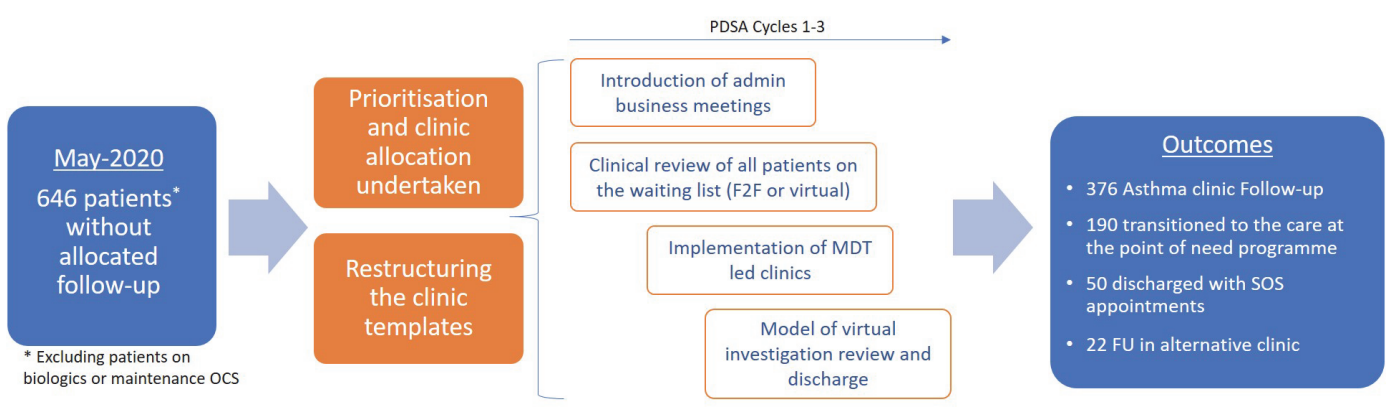

Abstract P154 Figure 1 A pathway transformation to transition from a 'routine' to a 'responsive' severe asthma service in the post COVID era

\section{P155 DELIVERING PHYSIOTHERAPY OUTPATIENT ASSESSMENT AND TREATMENT IN A SEVERE ASTHMA CLINIC IN THE ERA OF COVID-19}

C Mason, T Brown, R Harvey, B Reeves, S Folini, M Tolson, L Fox, L Wiffen, AJ Chauhan, R De Vos. Portsmouth Hospitals University NHS Trust, Portsmouth, UK

\subsection{6/thorax-2021-BTSabstracts.264}

Introduction and Objectives The global SARS-CoV-2 pandemic has forced clinicians to consider alternative methods of service provision to patients with respiratory conditions who were clinically vulnerable and/or advised to shield.

Breathing pattern disorders (BPD) are a common comorbidity affecting approximately one third of patients with asthma. The respiratory physiotherapy team within the Portsmouth Severe Asthma Service (PSAS) recognised the need to continue to assess and deliver treatment for patients with BPD particularly at a time of heightened anxiety.

Breathing retraining requires precise and highly specific assessment and treatment to ensure optimal outcomes and the decision to use a video conferencing platform was made to utilise the visual medium. To ensure quality service was being delivered, patients using the video conferencing platform to receive respiratory physiotherapy in the PSAS were asked for feedback.

Methods At the end of every video consultation, written feedback was requested. Sixty-nine responses were received from July 2020-May 2021. Patients were asked to rate their physiotherapy consultation from very good to very poor; how they would prefer to receive treatment; if they would use this method of consultation again and the ease of use of the video consultation.

Results Of the 69 responses:

- $68 / 69$ (98\%) would use the service again

- $58 / 69(84 \%)$ rated the service as very good

- 35/69 (51\%) would choose video over face to face appointments

- $23 / 69$ (33\%) would prefer to be seen face to face

- 63/69 (91\%) felt that accessibility of the video platform could be improved

Qualitative feedback was also gathered from patients and included statements such

- Excellent quality and a very thorough appointment.

- It was helpful to actually see a clinician face to face via video instead of a phone call
Conclusions Video consultations have proven to be a feasible and successful way of assessing BPD in asthma patients. Despite feedback regarding the ease of accessing the online platform being suboptimal, overarching positive responses to video consultations was received. With 51\% favouring being seen via video consultation rather than face to face, this has wider implications for patients and the NHS including reduced travel time to appointments and reduced waiting room pressures.

\section{P156 A REGIONAL STUDY OF THE AVAILABILITY, UPTAKE AND BARRIERS TO INHALER RECYCLING: PROMOTING ENVIRONMENTAL SUSTAINABILITY}

${ }^{1} \mathrm{~K}$ Liatsikos, ${ }^{2} \mathrm{R}$ Robinson, ${ }^{3} \mathrm{~A}$ Khashkhusha, ${ }^{1} \mathrm{~F}$ Shiham, ${ }^{2} \mathrm{H}$ Joplin, ${ }^{1} \mathrm{~A}$ Collins, ${ }^{2} \mathrm{H}$ Burhan. ${ }^{1}$ Liverpool School of Tropical Medicine, Liverpool, UK; ${ }^{2}$ Liverpool University Hospitals NHS Foundation Trust, Liverpool, UK; ${ }^{3}$ University of Liverpool, Liverpool, UK

\subsection{6/thorax-2021-BTSabstracts.265}

Introduction and Objectives Change is needed to reach the NHS environmental target to reduce inhaler carbon emissions by $50 \%$ over the next decade. We focused on inhaler sustainability, exploring available recycling schemes and community uptake. 73.000.000 inhalers are used annually in the UK and $63 \%$ form part of domestic waste. Our objective was to identify available recycling schemes nationally and regionally and explore factors influencing availability. Subsequently, to promote recycling schemes and increase local uptake.

Methods We performed an online search for available recycling schemes in the UK. Furthermore, we identified 21 pharmacies in Liverpool and surveyed them between March and May 2021. We aimed to determine how many inhalers they dispensed and whether they offered safe disposal and recycling. If they recycled, we explored what scheme they used and how they promoted it. If not, we explored why and what would encourage them to participate.

Results Following the end of the GSK 'completing the cycle' scheme in September 2020, there is one available scheme (TEVA One) nationally that has now paused enrolment. We received questionnaire responses from 14 of 21 pharmacies approached. On average, they dispensed 97.7 inhalers monthly. 64\% (9/14) accepted inhalers for safe disposal and $28 \%$ (4/14) reported accepting inhalers for recycling. However, on further investigation, this was for safe disposal only. Only $9.8 \%$ of inhalers dispensed were returned for safe 